\title{
El sentido de las cosas en la metáfora.
}

\author{
The meaning of things on the metaphor.
}

\author{
Kathya Jemio Arnez ${ }^{1}$ \\ Jemio A. Kathya. miradas Nº13 - 2015. ISSN: 0122 994X Págs 139 - 149 \\ Recepción: Mayo 6 de 2015 \\ Aprobación: Octubre 9 de 2015 \\ Publicación: Diciembre 17 de 2015
}

\section{Resumen}

Quien está en la búsqueda de herramientas para la construcción y revelación de significados ambiguos, ocultos o debajo de otros significados, muchas veces con el interés de ampliar el sentido, confundir la generalización con la especificidad de un concepto o desdibujar los límites de interpretación, se encuentra con la metáfora. Constituye un mecanismo para comprender y expresar conceptos complejos, sirviéndose de conocimientos elementales. Umberto Eco entiende que el lenguaje es por naturaleza y originariamente metafórico, por tanto toda regla surge con el fin de empobrecer el potencial metafórico que define al hombre como animal simbólico (Bustos 1999: 98). En su origen la metáfora es histórica porque su producción aparece en las sociedades y colectivos humanos que en el transcurso de su desarrollo y según sus circunstancias cotidianas materiales y culturales se apropian de la expresión, de esto resulta que los eventos cotidianos son fuente de su formación.

La lingüística cognitiva entiende que la metáfora produce y revela sentido mediante la superposición de conceptos: uno de estos escondido y otro expresivo. El primero es el que en su origen debía dar el sentido a las cosas, no obstante, el hablante superpone el segundo que pertenece a una familia de conceptos de categoría distinta y que, sin lógica aparente, llega al discurso y asume la tarea y logra, que la expresión adquiera un sentido nuevo, polisémico, metafórico y que manifieste una cosa en términos de otra.

La trasposición de los conceptos, de origen y de reemplazo muda, revela, ordena y estructura los sentidos de un lugar a otro y en orden diferente: son las prácticas, las creencias, los símbolos y los ritos, vinculados por la interacción social que son trastocados en su forma y sentido. Al respecto, Le Guern, (1985: 19) anota que desde esta perspectiva, la metáfora sale de la simple figura retórica: "Opera sobre la sustancia del lenguaje y no incide únicamente sobre la relación entre el lenguaje y la realidad expresada".

\footnotetext{
1 Profesora Asociada (PCJIC), Coordinadora de la Maestría en Comunicación Educativa (Convenio entre el PCJIC y la UTP), doctora en Historia (Universidad Nacional de Colombia), periodista (Universidad Carolina - Praga. República Checa) y Lingüista (UdeA). El artículo es resultado del empleo de la herramienta de análisis metafórico de la investigación de grado de la Maestría en Lingúística (2005). kajemio@elpoli.edu.co
} 
Se entiende que la metáfora no existe por sí misma, sino dentro y a través de una interpretación de la realidad, donde se crea una nueva significación (Ricoeur, 2001: 63).

Su empleo responde a diversas situaciones. En el lenguaje religioso es intermediación entre realidad trascendente e indecible y lo cotidiano. En esa intersignificación: Una metáfora llama a otras y engendra diversidad conceptual. El símbolo, dice Paul Ricoeur(2001:68), tiene cualidad de estabilidad, permanencia. Sólo es transformado, en su entrecruzamiento, la experiencia simbólica pide de la metáfora un trabajo de sentido.

Por todo lo anterior, la metáfora se concibe en este artículo como depositaria de conocimiento por cuanto amplía y estructura nuestro conocimiento del mundo y revela nuevas relaciones con los conceptos: en trasposición y con la particularidad de univocarse ${ }^{1}$, uno por o con el otro; de tal manera que el concepto meta determina el sentido y su realización, la ampliación o el cambio. La escuela cognitiva reconoce que "La metáfora es una forma de lenguaje figurativo donde un objeto se denota por otro por similitudes" (Renkema, 1999:135).

Esta condición respalda la afirmación de Anselm Strauss y Juliet Corbin (2002: 10), quienes se refieren a "la idea de que las personas actúan con una intención, [y a] la creencia de que la intención se define y se redefine con la interacción".

Las expresiones metafóricas contenidas en el texto son útiles en calidad de dispositivos de análisis ya que la unicidad de sentido que las distingue se logra de manera polisémica. Quienes se encuentran en la necesidad de develar sentidos, intenciones detrás del lenguaje formal, descubren el plurisignificado de los conceptos, la apariencia y la sutileza de mostrar y ocultar intenciones o expresarse metafóricamente.

En este trabajo se interpretan dos expresiones metafóricas y se describe su proceso de aparición en el lenguaje textual y simbólico.

Palabras Clave: Métafora, lenguaje, sentido, limites

\section{Abstract}

Who is in search of tools for building and disclosure of ambiguous meanings, hidden or under other meanings, often in the interest of broadening the sense, confusing widespread with the specificity of a concept or blur the boundaries of interpretation, meets metaphor. It provides a mechanism to understand and express complex concepts, using basic knowledge. Umberto Eco understands that language is originally metaphorical in nature and therefore every rule arises in order to impoverish the metaphorical potential that defines man as a symbolic animal (Bustos, 1999: 98). Originally metaphor is historical because their production appears in human societies and groups who in the course of its development and according to their material and cultural everyday circumstances appropriate the expression, It follows that everyday events are the source of their formation.

Cognitive linguistics understands that metaphor produces and reveals sense by overlapping concepts: one of these hidden and other expressive. The first is the one that originally had to give sense of things, however, the speaker overlaps the second belonging to a family of concepts of different categories and without apparent logic, comes to speech and assumes the task and succeeds, that the expression acquires a new meaning, polysemic, metaphoric and manifests one thing in terms of another. 
The transposition of the concepts, of origin and silent replacement, reveals, orders and structures the way from place to place and in a different order : are the practices , beliefs , symbols and rites, bound by social interaction that are disrupted in form and meaning. In this regard, Le Guern (1985:19) notes that from this perspective, the metaphor comes from the simple figure of speech: "It works on the substance of the language and It affects not only the relationship between language and reality expressed ".

It is understood that the metaphor does not exist by itself, but within and through an interpretation of reality, where a new meaning is created (Ricoeur, 2001:63).

Its use responds to various situations. In religious language is intermediation between the ineffable and trascendent reality and the everyday. In that intersignification: A metaphor calls to others and engenders conceptual diversity. The symbol, says Paul Ricoeur (2001:68), has the quality of stability, permanence. It is only transformed, in its crossing, symbolic experience asks metaphor a work of sense.

Given the above, the metaphor is conceived in this article as a depository of knowledge for how much it expands and structures our knowledge of the world and reveals new relationships with the concepts: In transposition and with the particularity of converge in the same direction, one by or with the other; such that the target concept determines the sense and its realization, the expansion or change. The cognitive school recognizes that "The metaphor is a form of figurative language where an object is denoted by other by similarities" (Renkema, 1999:135).

This condition supports the statement of Anselm Strauss and Juliet Corbin (2002:10), who refer to "the idea that people act with an intention, [ and ] the belief that the intention is defined and it redefines itself with interaction".

The metaphoric expressions in the text are useful as analytical devices because the uniqueness of meaning that distinguishes them is achieved in a polysemic way. Those in need of revealing senses, intentions behind the formal language, discover the multiple meanings of the concepts, appearance and subtlety of showing and hiding intentions or express metaphorically.

In this work two metaphoric expressions are interpreted and is described its emergence process in textual and symbolic language.

Key Words: Metaphor, language, meaning, limits

\section{Corpus}

Los datos que ingresan al análisis están conformados por expresiones metafóricas seleccionadas de un total de 533 codificadas y obtenidas de 40 artículos provenientes de una polémica ${ }^{2} \mathrm{y}$ publicados entre noviembre y diciembre de 1939 en los periódicos El Colombiano (EC), La Defensa (LD), El Diario (ED), El Heraldo de Antioquia(EH) y el semanario El Pueblo (EP). No se ingresó al análisis hemerográfico porque no es pertinente en este trabajo. Luego de la codificación se seleccionaron y clasificaron las metáforas de acuerdo con el método empleado.

El enfoque interaccionista presenta una perspectiva útil para nosotros en la teoría de Max Black. (Bustos, 1999: 104). Encuentra que es una forma de ver las cosas de diferente manera, lo que permite que la metáfora funcione como modelo y "revele" nuevas relaciones. Las cualidades que destaca y defiende son, la autonomía e irreductibilidad del sentido metafórico y la capacidad de constituirse en depositaria de conocimiento. Entiende que las 
unidades metafóricas son las oraciones, no las palabras, que constan de dos polos y se da tensión entre ellos. Los polos han de ser concebidos como sistemas, el significado de la metáfora surge de la interrelación entre los polos, el significado metafórico es irreductible y tiene contenido cognitivo. Encuentra finalmente, que el significado metafórico es producto de la interacción semántica entre estos dos polos denominados foco y marco.

Por su parte, E. Rivano, perteneciente a la escuela cognitiva californiana ${ }^{3}$ destaca la sistematicidad de los conceptos metafóricos que se relaciona a la naturaleza metafórica de nuestras actividades. En Las metáforas de la vida cotidiana, Lakoff y Johnson (1998: 85) entienden que éstas "...estructuran el sistema conceptual ordinario de nuestra cultura que se refleja en el lenguaje cotidiano". Presentada de esa forma, traduce nuestras creencias, sentimientos y valores que conforman nuestra conciencia individual y colectiva. Escriben, "las metáforas estructuran parcialmente los conceptos que usamos y esta estructura se refleja en nuestro lenguaje literal".

Este enfoque distingue y define las categorías sin perder de vista que resultan de atributos básicos del conocimiento social y - observan Susan Condor y Charles Antaki (2000: 453-457) — su limitación es la sobregeneralización e inexactitud. Surgen de nuestra interacción directa (categorías que emergen de la experiencia física directa, por ejemplo, relativos al ambiente en que nos movemos y sus direcciones y dimensiones) e indirecta (categorías que emergen de conceptos abstractos). Tres teorías cognitivas sobre las categorías son empleadas en la presente investigación: La teoría de los prototipos que reconoce niveles básicos y niveles periféricos de categorización, la teoría de las semejanzas de familia que define conceptos afines con sólo posibilidades asociativas, por último, la teoría del nivel básico, en la que Rosch (Cuenca y Hilferty, 1999: 48) propuso tres niveles de organización: De éstos nos interesan, el superordinado (que incluye miembros de gran diversidad, "categoría parasitaria") y el nivel básico que es el más concreto y preciso que integra los atributos esenciales categoriales.

Cabe anotar que las categorizaciones en lingüística cognitiva son comprendidas como flexibles, "los prototipos y las fronteras categoriales pueden cambiar en un contexto particular y dependen de nuestro conocimiento social y cultural, que se organiza en modelos cognitivos (de naturaleza psicológica e individual) y culturales (de naturaleza social y colectiva).

El problema esencial de las teorías cognitivas son las representaciones cognitivas y sus procesos que van del mundo externo al mundo interior y viceversa con la consecuente dialéctica de cambio. Estas representaciones incluyen palabras, signos, imágenes y conceptos. Puede existir un carácter literal o no y gracias a las inferencias que podemos hacer encontramos que sistemas conceptuales que conforman polos autónomos tienen algo en común (fuerza, tensora), que crea un nuevo concepto. Entienden Graesser, Morton A. et al. (1999: 418), que las representaciones semánticas que construimos "están ancladas en un rico cuerpo de experiencias y conocimiento general del mundo (que varía de una persona a otra). Al mismo tiempo, las representaciones semánticas son por lo general fragmentarias (en lugar de completas), vagas (en lugar de precisas), redundantes, abiertas y esquemáticas. Y sin embargo, a pesar de todas estas aparentes indefiniciones del sistema, los escritores/ hablantes consiguen construir mensajes que, por lo general, los lectores/oyentes 
pueden recuperar con impresionante precisión".

Interesa el análisis de la conformación lingüística y cultural de metáforas detrás de las expresiones de dos metáforas dicotómicas: peso y luz.

\section{Ordenamiento conceptual}

Los datos de la investigación en su calidad de expresiones metafóricas son categorizados de acuerdo a su naturaleza, propiedades y dimensiones. Lakoff y Johnson, encuentran tipos de metáforas. Por ejemplo las de orientación y las ontológicas que organizan un sistema global de conceptos con relación a otros: dentro/fuera, delante/atrás, etc. Funcionan con una lógica de ubicación constante, de manera que su interpretación cultural recupera esas mismas cualidades. Así todo lo que está arriba, coincide con lo feliz, lo bueno, ascenso, etc. En sentido contrario, las categorías conceptuales negativas se ubican hacia abajo y coinciden con malo, feo, descenso, negro, etc. La coherencia entre la orientación y la representación cultural de la expresión metafórica se presentan en algunos ejes culturales que empalman con este criterio. Se distinguen los casos que hacen uso de expresiones como alto, elevarse, hondo, hunden, levanten, duro, aplanado, inflaciones, para referirse a conceptos de mayor abstracción: ideología, posición laboral, problema, política, y que se corresponden conceptualmente entre sí: alto cargo (E.P., 4), No puede elevarse a la categoría de problema de estado porque es una nimia cuestión (E. C., 2), el hondo sabor ideológico (E.P., 4), hunden a la política su lenguaje (E. C., 18).

Se obtienen las expresiones con componente metafórico en una lectura atenta del discurso objeto del estudio. En ese proceso se advierte la presencia de términos o conceptos que por sí solos sin el contexto son extraños al discurso. Esta fase del trabajo se realiza de manera preteórica. En esta primera etapa del proceso se hallan aspectos principales del análisis, es "la interacción que tienen lugar entre los datos y el investigador en el proceso de recolección y análisis de los mismos" (Strauss A. y Juliet Corbin, p.64).

\section{Estructura de expresiones metafóricas}

Cada metáfora ubica una base física y cultural en la que se registra el proceso de apareamiento de dominios conceptuales, de modo que una expresión metafórica (palabra, frase, oración) es una realización (superficial) de este entramado de correspondencias conceptuales.

Así encontramos las siguientes expresiones organizadas por categorías a la que corresponden conceptos próximos, denominados también familiares, mencionados anteriormente.

\begin{tabular}{|l|l|}
\hline Categoría & Conceptos familiares a la categoría \\
\hline Limpio & Aseo, pulcritud, honrado, decente \\
\hline Puro & Simple, sin mezcla \\
\hline Bueno & $\begin{array}{l}\text { Gustoso, sano, no deteriorado, suficiente, } \\
\text { bondad }\end{array}$ \\
\hline Claro & $\begin{array}{l}\text { Limpio, puro, cristalino, diáfano, } \\
\text { inteligible, sincero }\end{array}$ \\
\hline Luz & Energía, claridad, lumbre, inteligencia \\
\hline Color & Sustancia, matiz, físico \\
\hline
\end{tabular}

La tabla derivó de algunas de las expresiones encontradas en el corpus codificado:

brecha luminosa en persecución, (E. C., 10)

blasón de la más pura ortodoxia, (E. P., 3)

noble pureza de su vida, (E.D., 6)

Ha ruborizado a algunas personas, (E. C., 8)

atacándome una gris mayoría, (E. C., 12)

mujer juego de líneas, colores, oscuridades, (E. H.,2)

El menor sonrojo, (E. C., 6) 
demuestra a las claras, (E.P., 4)

esclarecidos miembros, (E. C., 5)

se nos habia difuminado del paisaje, (E. C., 8)

momentos lúcidos, (E. C., 13)

se ha visto claro el juego de los taumaturgos de la intriga, (E.D., 7)

despejarse una conciencia, (E. C., 18)

puramente subjetivo, (E.D., 1)

Poseen una conciencia caótica y borrosa, (E. C., 18)

Declaro desierto el certamen (E.C., 12)

El conjunto nos orienta a la distinción cultural de la metáfora. En el discurso se descubre la sistematicidad de estos conceptos, dirigidos bajo el mismo eje cultural. Habitualmente el orden (abajo, sucio, pesado, etc.) se ubica abajo y viceversa. El caso siguiente ilustra, a partir de una tabla, la alineación de conceptos hacia un mismo fin: LAS BUENAS COSTUMBRES SON LIMPIAS, SON PURAS, SON CLARAS, SON ARRIBA (orientación cultural en la designación de bueno, positivo), lo que finalmente lleva a verbalizar: LA MORAL ES LUZ que, además de lo señalado, permite dilucidar sobre la fuerza católica en la moral por el plurisignificado de luz en la religión. En la metáfora LA MORAL ES LUZ se presenta el caso de un mismo dominio meta, (luz) tomado en su variación lingüística de diversos dominios de origen, (limpio, claro, puro, lozano), conceptos con propiedades inherentes a limpieza, claridad, pureza y lozanía que se integran a las propiedades de luz. Esto confirma la coherencia entre metáforas y conceptos.

Observaciones: Limpio, puro, bueno, claro, luz, orientación, corresponden a distintas categorías conceptuales relacionándose, por una parte, las categorías puro, limpio y color, y por otra parte, el cuerpo y los valores estéticos (bello/feo) y el alimento. Esta relación se sostiene en expresiones metafóricas que remiten a: la orientación (asignación de atributos culturales al desplazamiento hacia arriba o hacia abajo), el movimiento (ya sea camino, trayecto o sólo proceso), el estado (momentos) y el tiempo (lapso en que transcurre el momento). La metáfora: MORAL ES LUZ. Veamos las semejanzas en los atributos particulares:

\begin{tabular}{|c|c|c|}
\hline Categoría & Ejemplos & dominio meta \\
\hline \multirow[b]{2}{*}{$\begin{array}{l}\text { L i m p i o / } \\
\text { sucio }\end{array}$} & $\begin{array}{l}\text { tanta falta hace la higiene } \\
\text { moral (L.D., l) }\end{array}$ & \multirow{3}{*}{$\begin{array}{l}\text { LAS BUENAS } \\
\text { COSTUMBRES SON } \\
\text { LIMPIAS } \\
\text { LAS BUENAS } \\
\text { COSTUMBRES SON } \\
\text { PURAS }\end{array}$} \\
\hline & $\begin{array}{l}\text { Mire el arte con mente } \\
\text { limpia (E.D., I) }\end{array}$ & \\
\hline \multirow[t]{2}{*}{ Pureza } & $\begin{array}{l}\text { Moderno horno crematorio } \\
\text { que todo lo higieniza y } \\
\text { purifica (L.D., l) }\end{array}$ & \\
\hline & $\begin{array}{l}\text { noble pureza de su vida } \\
\text { (E.D., 4) }\end{array}$ & \multirow{2}{*}{$\begin{array}{l}\text { LAS BUENAS } \\
\text { COSTUMBRES SON } \\
\text { CLARAS }\end{array}$} \\
\hline \multirow{3}{*}{ Claridad/luz } & $\begin{array}{l}\text { Esclarecidos miembros (E. } \\
\text { C., 5) }\end{array}$ & \\
\hline & $\begin{array}{l}\text { momentos lúcidos (E. C., } \\
13 \text { ) }\end{array}$ & $\begin{array}{l}\text { LAS BUENAS } \\
\text { COSTUMBRES SON } \\
\text { LUZ }\end{array}$ \\
\hline & $\begin{array}{l}\text { Despejarse una } \\
\text { conciencia (E.C., 18) }\end{array}$ & \multirow{2}{*}{$\begin{array}{l}\text { LAS BUENAS } \\
\text { COSTUMBRES SON } \\
\text { ARRIBA }\end{array}$} \\
\hline Color & $\begin{array}{l}\text { Atacándome una gris } \\
\text { mayoría }(E . C ., 12)\end{array}$ & \\
\hline Alimento & $\begin{array}{l}\text { asoma lozano y sazonado } \\
\text { (E. C., 18) }\end{array}$ & $\begin{array}{l}\text { LAS BUENAS } \\
\text { COSTUMBRES SON } \\
\text { BLANCAS } \\
\text { LAS BUENAS } \\
\text { COSTUMBRES SON } \\
\text { ALIMENTO }\end{array}$ \\
\hline
\end{tabular}

Correspondencias conceptuales y de sentido en la metáfora la piedra de escándalo

Este segundo caso está vinculado a metáforas construidas con categorías conceptuales que habitualmente extraemos de la experiencia física y perceptual: peso, volumen, hacia abajo y son llevados además hacia un lado, empleado regularmente para conseguir una valoración negativa. 


\section{VOLUMEN - PESO}

la piedra de escándalo (E.D., 1)

A tomado a su cargo, (E. C., 14),

un ligero comentario, (E. C., 8),

ese grande ideal, (E.P., 4),

merece una gran carcajada, (E.D., 3),

Nuestro grueso publico, (E.D., 1),

alma diminuta, (E. C., 13),

cuando descargamos unas frases, (E.D., 8)

recuperado su perdida gravedad (E. C., 8)

El Pueblo aguanta esperpentos (E.D., 5)

Aquellos que si resisten (L.D., 2);

De manera general las correspondencias de los conceptos se refieren a la asociación, semejanza y relación que tienen en un dominio de la experiencia en términos de otro porque uno de los significados se superpone al del otro. E. Rivano anota sobre este aspecto que: "El sistema metafórico, centro de nuestro sistema conceptual establece relaciones conceptuales que subyacen en las expresiones, relaciones que llevan a generalizaciones con validez empírica"4. En la expresión siguiente se identifican conceptos, que en su origen se comprenden bajo el término piedra en la escena de un escándalo. El sentido de piedra en el contexto sólo puede ser entendido como motivo de relaciones problemáticas, es decir que los escándalos son producidos por un tipo de fenómeno que tiene las propiedades de una piedra (carga, peso, volumen) y su uso podría estar relacionado a contextos sociales problemáticos o violentos, o en general, entendemos que todo problema es una carga, un peso, (dominio conceptual meta). De esta inferencia resulta la metáfora Los PROBLEMAS SON PIEDRAS (¡QUÉ PIEDRA!).
No obstante a lo anterior se agrega la coherencia cultural con la existencia en el discurso de gran cantidad de expresiones similares es decir, diversos y amplios dominios conceptuales o con semejanzas familiares son partícipes del apareamiento.

Dominios CONCEPTUALES de crigen varios y un dominio conceptual meta
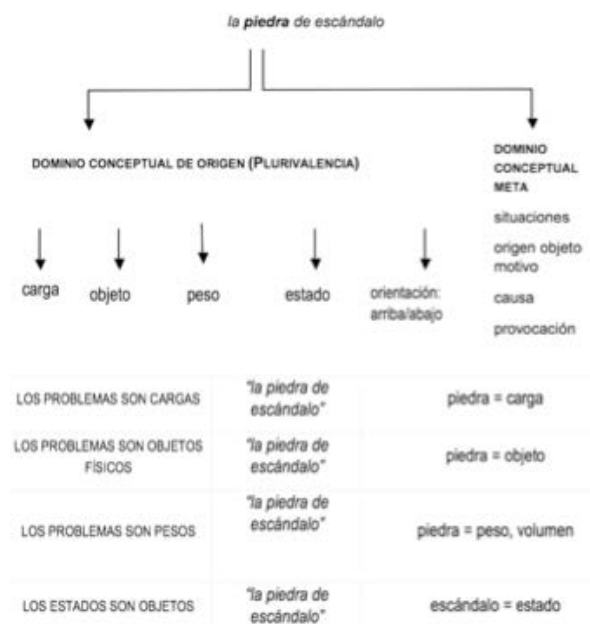

Una metáfora como LOS PROBLEMAS SON PIEDRAS organiza la expresión de acuerdo con el apareamiento conceptual piedras/ problemas. Cuando se conceptualiza piedra se infiere que es una carga, un peso; al contrario de la liviandad, facilidad, "ligero", leve, de poco peso, por tanto, de acuerdo a la inferencia de peso/problema, el caso de ligero tiene semejanza a poco problemático, sin dejar de entender que la liviandad del comentario atraviesa por una percepción negativa (un ligero comentario). Por otro lado, el problema es algo malo, negativo en su valoración. Esto se ajusta a la orientación que los conceptos adquieren en nuestra cultura, donde resulta que lo bueno y lo malo, culturalmente se representan en un eje con dirección espacial opuesta. Lo bueno, lo bello, el bien, lo positivo, etc., depositamos en lo alto, arriba. Una situación distinta, presenta lo negativo, lo malo, los problemas, las cargas que depositamos en el lado opuesto, lo que se ubica abajo, junto a lo negativo, lo feo, lo pesado (piedra). 
Esta tercera inferencia permite además encontrar que la metáfora LOS PROBLEMAS SON PIEDRAS puede ser nombrada también cOMO LOS PROBLEMAS SON ABAJO y resulta una metáfora orientacional que coincide con las direcciones, espacios, distancias de nuestras valoraciones culturales.

Con estas expresiones consideramos las situaciones experienciales de "problema" en términos de peso, resistencia, volumen. Es decir, las categorizamos como cargas. Es entonces donde se advierte que el dominio conceptual meta, piedra, está reemplazando a problema. Por otra parte, las correspondencias que se encuentran entre piedra y carga en asociación a la polémica o a los problemas, permiten mapear la ontología del dominio de origen (piedra) en la ontología meta (carga, escollo). Aclaro que cuando nombramos la metáfora en su variedad: Los PROBLEMAS SON CARGAS, sólo estamos dando nombre al apareamiento.

La expresión: la piedra de escándalo presenta una cantidad de elementos que ingresan a los conceptos de contexto, significado, escena y propiedades. Observemos:

En su significado, la expresión ha sido empleada en situaciones problemáticas. Las propiedades de una piedra, remite a cargas, pesos, objetos físicos, orientación cultural negativa, abajo, y todas estas remisiones están unidas al escándalo: la situación que es agravada por una circunstancia negativa de peso, volumen y otros conceptos similares.

La escena se asocia al peso, provocación, carga, problema, escollo, vinculado a nuestra experiencia directa. Podemos remontarnos a la función moral y de escarmiento que tenía ésta en la Antigüedad, al momento de condenar. Recordemos las piedras con que sepultaban a las mujeres infieles en las sociedades antiguas, que además se asemeja al sentido de piedra en, "lanzó la piedra y ocultó la mano".

En la fabricación de sentido la piedra en el contexto de escándalo y al interior del discurso analizado tiene un sentido orientacional hacia abajo y establece valores. En este caso a lo físico, pesado, carga, grave.

Consecuentemente se puede clasificar las expresiones metafóricas en su concepción perceptual, como objeto, proceso, estado y se encontrara categorías conceptuales que derivan de adelante/atrás, adentro/afuera, cerca/lejos, visible/oculto, objeto bello/ feo, frío/caliente, dirección, movimiento, bueno/malo, cantidad/calidad,limpio/ sucio, sano/ enfermo. Es decir, la expresión metafórica se reconoce también por su transversalización de sentido que deriva de categorías conceptuales distintas y variadas. Son categorías que hacen alusión a categorías conceptuales perceptuales, de estado, temperatura, proceso, elementos físicos (objetos). Veamos en la tabla siguiente: 


\section{Categorías conceptuales experienciales, directas e indirectas}

PERCEPTUAL, OBJETO, PROCESO, ESTADO

EXPRESIONES METAFÓRICAS CON REFERENCIA HEMEROGRÁFICA CODIFICADA

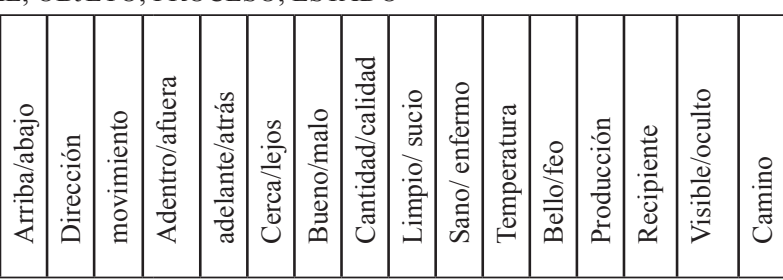

Luz/oscuridad, Volumen/peso

\begin{tabular}{|l|l|l|l|l|l|l|l|l|l|l|l|l|l|l|l|l|l|}
\hline (E. C., 6) & "Más de un juicio colérico" & & & & & & & & $\mathrm{X}$ & & & $\mathrm{X}$ & & & & & \\
\hline (E. C., 6$)$ & El menor sonrojo & & & & & & & & $\mathrm{X}$ & & & & & & & & \\
\hline (E. C., 8$)$ & Ha ruborizado a algunas personas & & & & & & & & & & & $\mathrm{X}$ & & & & & \\
\hline (E. C., 8$)$ & $\begin{array}{l}\text { tratan de abrir una brecha luminosa en } \\
\text { persecucion }\end{array}$ & & $\mathrm{X}$ & & $\mathrm{X}$ & $\mathrm{X}$ & & & & & & & & & & & $\mathrm{X}$ \\
\hline
\end{tabular}

\begin{tabular}{l|l}
\hline (E.D., 3) & muchos tartufos puritanos entienden
\end{tabular}

(E. C., 18) $\quad$ asoma lozano y sazonado

(E. C., 18) Despejarse una conciencia

\begin{tabular}{|l|l}
\hline (E.D., 3) & reciben luz \\
\hline$(E . C ., 8)$ & se nos habia difuminado del paisa \\
\hline
\end{tabular}

\begin{tabular}{|l|l}
\hline (E.D., 4) & noble pureza de su vida \\
\hline
\end{tabular}

\begin{tabular}{l|l} 
(E.D., l) & Puramente subjetivo
\end{tabular}

(E. H.,4) mujer juego de líneas, colores, oscuridades

\begin{tabular}{l|l}
\hline (E. C., 5) & esclarecidos miembros \\
\hline
\end{tabular}

(E. C., 12) atacándome una gris mayoría

(E. C., 13) momentos lúcidos

(L.D., 1) Moderno horno crematorio que todo lo

\begin{tabular}{|l|l} 
& higieniza y purifica \\
\hline (L.D., I) & "civismo e higiene"
\end{tabular}

\begin{tabular}{|l|l}
\hline (L.D., I) & "tanta falta hace la higiene moral" \\
\hline
\end{tabular}

\begin{tabular}{l|l} 
(L.D., l) & junta de higiene
\end{tabular}

(E.D., 4) Hay que poseer higiene mental

\begin{tabular}{l|l}
\hline (L.D., l) & $\begin{array}{l}\text { Moderno horno crematorio que todo lo } \\
\text { higieniza y purifica }\end{array}$
\end{tabular}

\begin{tabular}{l|l}
\hline (E.P., 4) & demuestra a las claras \\
\hline
\end{tabular}

(E. C., 12) declaro desierto el certamen

(E.D., 5) $\quad \begin{aligned} & \text { se ha visto claro el juego de los taumaturgos } \\ & \text { de la intriga }\end{aligned}$

\begin{tabular}{l|l} 
(E.D., 1) & nuestro grueso publico
\end{tabular}

\begin{tabular}{l|l}
\hline (L.D., 2) & Aquellos que si resisten
\end{tabular}

\begin{tabular}{l|l} 
(E.D., l) la piedra de escándalo &
\end{tabular}

(E. C., 13) ruindad de alma diminuta

\begin{tabular}{|l|l}
\hline (E.D., 6) & cuando descargamos unas frases
\end{tabular}

\begin{tabular}{l|l} 
(E. C., 8) & Recuperado su perdida gravedad
\end{tabular}

\begin{tabular}{l|l} 
(E.D., 5) & El pueblo aguanta esperpentos
\end{tabular}

(E. C., 14) A tomado a su cargo

\begin{tabular}{|l|l}
\hline (E.D., 2) & merece una gran carcajada
\end{tabular}

(E. C., 8). $\quad$ un ligero comentario

\begin{tabular}{l|l}
$(E . P ., 4)$ & ese grande dieal
\end{tabular} 


\section{Conclusiones}

En el modelo de análisis la relación de apareamiento entre dominios suele presentarse de múltiples formas. Podemos hallar diversos dominios de origen (en cantidad y origen), sostenidos por coherencia conceptual con un mismo dominio meta. Así, piedra tiene equivalencia a carga, objeto, estado, volumen y "hacia abajo" en su orientación. Lo mismo sucede con LA MORAL ES LUZ.

Las metáforas inicialmente inconexas entre sí, agrupadas en grandes núcleos comunes se asocia a binomios conceptuales: cantidad/calidad, dentro/fuera, arriba/ abajo, visible/oculto, Adelante/atrás, Parte/todo, claro/oscuro, volumen/peso. Lo que revela regularidad luego de su agrupamiento en una red de metáforas comunes y su sistematicidad con aspectos comunes sobresalientes que aluden, entre otros, a una base física y cultural.

El tejido conceptual del que deriva la metáfora se pudo encontrar con la transversalización de categorías y conceptos. Por un lado conceptos que derivan de claridad, oscuridad y otros derivan de volumen.

Las valoraciones culturales depositadas no son uniformes y determinantes.

\section{Referencia Bibliográfica}

Bustos Guadaño de, E. (1999). "Metáfora”. En: Dascal, Marcelo (comp.), Filosofía del Lenguaje II. Pragmática. Enciclopedia Iberoamericana de filosofía. Valladolid: Trotta.

Calvo, T. y Remedios, A. (1987). Paul Ricouer: Los caminos de la interpretación. España: Antrhopos.
Corral, A. (1999) Los aspectos implicados en la comprensión de las metáforas. En: Revista Cultura y Educación, Universidad de Salamanca.

Cuenca, J. y Hilferty, J. (1999). Introducción a la lingüística cognitiva. Barcelona: Ariel.

Ducrot, O. (1982). Ilocutorio y performativo. Decir y no decir. Barcelona: Anagrama.

Eggins, S. y Martín, J. (2000). "Géneros y registros del discurso". En: Teun Van Dijk, El discurso como estructura y proceso (trad. Perla Wagner). Barcelona: Gedisa.

Escandel Vidal, M. (1993). Introducción a la pragmática. Madrid: Anthropos-Uned.

Fajardo, L. (2001) La metáfora: ¿un recurso cognoscitivo o un recurso estilístico? En: Revista de la Asociación de Exalumnos del Seminario Andrés Bello, 10.

García, J. (2000). Informar y narrar: el análisis de los discursos en las investigaciones de campo. En: Revista de Antropología Social, 9.

Grice, H. (1982). "La lógica y la conversación”. Lenguaje y Sociedad. Cali: Traducciones Univalle.

Haidar, J. (1998). Análisis del discurso. En: Jesús Galindo Cáceres (coord.), Técnicas de investigación social en Sociedad, cultura y comunicación. México: Addison Wesley Longman.

Horn, L. (1990). “Teoría pragmática". En: Frederick Newmeyer (comp.). Panorama de la lingüística moderna I. Madrid: Universidad de Cambridge.

Kleiber, G. (1995). Semántica de los prototipos. Madrid: Visor Libros.

Lakoff, G. y Johnson, M. (1998). Metáforas de la vida cotidiana. Madrid: Catedra. Cuarta edición. Colección Teorema.

Le Guern, M. (1985). La metáfora y la metonimia. Madrid: Cátedra. Cuarta edición. 
Maingueneau, D. (1996). "L'analyse du discours en France aujourd'hui". Le français dans le monde. Recherches et applications. Le discours : enjeux et perspectives. France: Hachette, numéro especial, juillet.

Osorio, J. (2001). La conceptualización del control en el español de Chile: un análisis metafórico. En: Revista de Lingüística teórica y aplicada, 39.

Pinto, M. (2001). Lenguaje y cognición: del lenguaje científico al mundo olvidado de las sensaciones. En: Revista Educación y Pedagogía, 13(31).

Renkema, J. (1999). Introducción a los estudios sobre el discurso. Barcelona: Gedisa.

Ricoeur, P. (2001). "El Lenguaje como discurso". En: Paul Ricoeur, Teoría de la interpretación (trad. Graciela Monges Nicolau). México: Siglo XXI. Cuarta edición.

Ricoeur, P. (2001). Teoría de la interpretación. México: Siglo XXI.

Ricoeur, P. (1998). ¿Qué es un texto? Lingüística y Literatura, 33, 86-105.

Wodak, R. y Meyer, M. (2003): Métodos de análisis crítico del discurso. Barcelona: Gedisa.

Searle, J. (1982). ¿Qué es un acto de habla? Lenguaje y Sociedad, 79-99.

Searle, J. (1980[1969]). Actos de habla. Madrid: Cátedra.

Sola, P. (2001). "Léxico e ideología en Les cloches de Bâle". En: Isabel Uzcanga et al., Presencia y renovación de la lingüística francesa. Salamanca: Universidad de Salamanca.

Rivano, E. (1997). Metáfora y Lingüística Cognitiva. Santiago: Bravo y Allende Editores.

Valladares, S. (2000). De cómo sacar partido a las palabras. En: Revista de Antropología Social, 9.

\section{Notas al lector}

1 Rae. Convenir en una razón misma dos o más cosas. NÚÑEZ DE TABOADA, M. Diccionario de la lengua castellana, para cuya composición se han consultado los mejores vocabularios de esta lengua y el de la Real Academia Española, últimamente publicado en 1822; aumentado con más de 5000 voces o artículos que no se hallan en ninguno de ellos, 2 vols. París, Seguin, 1825. Reproducido a partir del ejemplar de la Biblioteca Nacional de Madrid, 1/16871 - 1/16872

2 La polémica periodística se originó en 1939 y continuó durante algunas décadas a causa de la exposición pictórica, en la que Débora Arango presentó de dos desnudos, en el Club Unión de Medellín (1939).

3 Emilio Rivano, Metáfora y Lingüística Cognitiva, Emilio Rivano, 1997, Santiago: Bravo y Allende Editores

4 Rivano, Emilio., Op. cit., p. 5. 